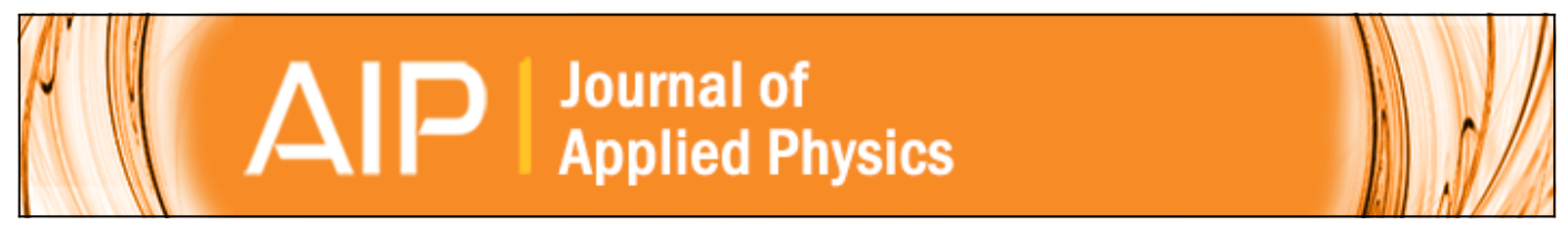

\title{
Crystal size and oxygen segregation for polycrystalline GaN
}

K. S. A. Butcher, H. Timmers, Afifuddin, Patrick P.-T. Chen, T. D. M. Weijers, E. M. Goldys, T. L. Tansley, R. G. Elliman, and J. A. Freitas Jr.

Citation: Journal of Applied Physics 92, 3397 (2002); doi: 10.1063/1.1499232

View online: http://dx.doi.org/10.1063/1.1499232

View Table of Contents: http://scitation.aip.org/content/aip/journal/jap/92/6?ver=pdfcov

Published by the AIP Publishing

\section{Articles you may be interested in}

Anisotropy of tensile stresses and cracking in nonbasal plane Al x Ga $1-x$ N / GaN heterostructures

Appl. Phys. Lett. 96, 041913 (2010); 10.1063/1.3276561

Structural properties of wurtzitelike ScGaN films grown by NH 3 -molecular beam epitaxy

J. Appl. Phys. 106, 113533 (2009); 10.1063/1.3268466

Anisotropic strain relaxation in a -plane GaN quantum dots

J. Appl. Phys. 101, 063541 (2007); 10.1063/1.2713937

Effects of grain size on the mosaic tilt and twist in InN films grown on GaN by metal-organic chemical vapor deposition

Appl. Phys. Lett. 89, 092114 (2006); 10.1063/1.2345224

Local conductivity and surface photovoltage variations due to magnesium segregation in p-type GaN

J. Appl. Phys. 95, 6225 (2004); 10.1063/1.1713025

\section{AIP $\left.\right|_{\text {APL Photonics }}$}

APL Photonics is pleased to announce Benjamin Eggleton as its Editor-in-Chief

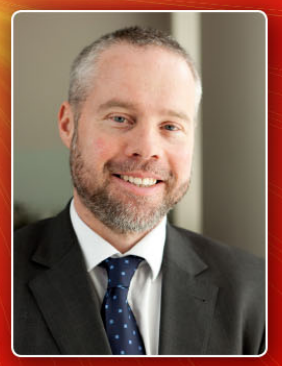




\title{
Crystal size and oxygen segregation for polycrystalline GaN
}

\author{
K. S. A. Butcher ${ }^{\mathrm{a})}$ \\ Semiconductor Science and Technology Laboratories, Department of Physics, Macquarie University, \\ Sydney NSW 2109, Australia \\ H. Timmers ${ }^{\text {b) }}$ \\ Department of Electronic Materials, Research School of Physical Sciences and Engineering, \\ Australian National University, Canberra, ACT 0200, Australia
}

Afifuddin and Patrick P.-T. Chen

Semiconductor Science and Technology Laboratories, Department of Physics, Macquarie University, Sydney NSW 2109, Australia

T. D. M. Weijers ${ }^{\text {b) }}$

Department of Electronic Materials, Research School of Physical Sciences and Engineering, Australian National University, Canberra, ACT 0200, Australia

E. M. Goldys and T. L. Tansley

Semiconductor Science and Technology Laboratories, Department of Physics, Macquarie University, Sydney NSW 2109, Australia

R. G. Elliman

Department of Electronic Materials, Research School of Physical Sciences and Engineering, Australian National University, Canberra, ACT 0200, Australia

J. A. Freitas, Jr.

ESTD-Electronic Materials Branch, U.S. Naval Research Laboratory, 4555 Overlook Ave.,

S. W. Washington, D.C. 20375-5347

(Received 13 May 2002; accepted for publication 13 June 2002)

The grain size for polycrystalline GaN, grown in low-temperature gallium-rich conditions, is shown to be correlated to the oxygen content of the films. Films with lower oxygen content were observed to have larger crystals with an increased tendency to a single-preferred crystal orientation. Elastic recoil detection analysis with heavy ions (i.e., $200 \mathrm{MeV}{ }^{197} \mathrm{Au}$ ions) was used to determine the composition of the GaN films grown for the study, including the hydrogen, carbon, gallium, nitrogen, and oxygen content. Atomic force microscopy and x-ray diffraction were used to study the sample morphology. From these measurements, the available surface area of the films was found to be sufficient for a significant proportion of the oxygen present in the films to segregate at the grain boundaries. This interpretation is consistent with earlier theoretical studies of the formation and segregation of the $V_{\mathrm{Ga}^{-}}\left(\mathrm{O}_{\mathrm{N}}\right)_{3}$ defect complex at dislocation sites in gallium-rich $\mathrm{GaN}$. For this work, however, the defect complex is believed to segregate at the grain boundary of the polycrystalline GaN. (C) 2002 American Institute of Physics. [DOI: 10.1063/1.1499232]

\section{INTRODUCTION}

Present gallium nitride device technology is based on what in the literature is nominally described as single crystal material grown at temperatures generally above $1000^{\circ} \mathrm{C}$, on sapphire or $\mathrm{SiC}$ substrates. ${ }^{1}$ However, these substrate materials tend to be expensive so that the cost effective application of present devices is limited. Growth at high temperatures also entails a large expenditure in temperature resistant growth equipment and ancillaries. Considerable savings and expansion in device use can potentially be achieved by lower-temperature growth, say below $650^{\circ} \mathrm{C}$, on less expen-

\footnotetext{
a) Author to whom all correspondence should be addressed; electronic mail: sbutcher@ics.mq.edu.au

b) Also at: Department of Nuclear Physics, Research School of Physical Sciences and Engineering, Australian National University, Canberra, ACT 0200, Australia.
}

sive substrate materials. At present, material quality is lower at such low growth temperatures, and polycrystalline material is prevalent when growing on low cost, temperature sensitive substrates, such as glass or silicon. However, in many applications, polycrystalline material may be of sufficient quality for device use. There has also been much recent improvement in the quality of polycrystalline material. For example, Bour et $\mathrm{al}^{2}$ have demonstrated a blue light-emitting diode fabricated from polycrystalline GaN grown on quartz using a GaN buffer layer. Despite its potential, interest in polycrystalline $\mathrm{GaN}$ is small in comparison to that of singlecrystal material, and the knowledge base for this material is also correspondingly small. Material grown below $650{ }^{\circ} \mathrm{C}$ tends to be gallium rich because of the poor desorption of free gallium. ${ }^{3-5}$ Therefore, the wider utilization of this material is closely related to understanding the defect structure of gallium-rich $\mathrm{GaN}$. 
For this work, we have varied the oxygen content of polycrystalline $\mathrm{GaN}$ films grown under otherwise similar conditions, and examined the changes in film morphology using atomic force microscopy (AFM) and x-ray diffraction (XRD). The films were grown under gallium-rich conditions. A system for elastic recoil detection (ERD) analysis with heavy ion beams, developed at the Australian National University (ANU), ${ }^{6-8}$ was used to determine the elemental composition of the films. In the following section, some background is given into the issue of oxygen incorporation in gallium-rich GaN. Experimental results for the ERD and morphology studies are then presented, and finally the results are discussed in the context of polycrystalline GaN.

\section{BACKGROUND}

Recent work in literature suggests that oxygen acts as a shallow dopant in GaN and may be partially responsible for the unintentional $n$-type background doping of this material. ${ }^{9,10}$ However, growth conditions can play a strong role in determining how a foreign atom incorporates in a host matrix. For instance, oxygen is known to take part in the formation of point defects for arsenic-rich GaAs grown by molecular-beam epitaxy (MBE) or metalorganic chemical vapor deposition. ${ }^{11}$ However, in GaAs grown by liquid phase epitaxy (LPE), under gallium-rich conditions, oxygen behaves as a shallow donor. ${ }^{12}$ As a result of this the deep level trap density is suppressed in LPE GaAs and the minority carrier diffusion length - a measure of material purity—can be several orders of magnitude higher than for arsenic-rich GaAs. ${ }^{13}$

Gallium-rich GaN is easily obtained at temperatures below $750{ }^{\circ} \mathrm{C}$ because below that temperature, free gallium has an extremely low desorption rate. ${ }^{3-5} \mathrm{GaN}$ buffer layers are commonly grown under Ga-rich conditions, since the presence of excess gallium in the buffer layer improves the buffer layer morphology and has led to an improvement in the photoluminescence of subsequently deposited layers. ${ }^{14}$ Gallium-rich growth conditions are also easily achieved in MBE growth where surface accumulations of free Ga have been observed under low temperature conditions. ${ }^{5}$ In fact, slightly Ga-rich conditions, below $750{ }^{\circ} \mathrm{C}$, are often sought because of the improved morphology of the layers. ${ }^{3,10}$ There is, therefore, considerable interest in Ga-rich GaN.

In this article, we consider the role of oxygen in a specific circumstance, that is, the role of oxygen in gallium-rich polycrystalline $\mathrm{GaN}$. Based on experience gained from the example of GaAs, in GaN, we should expect oxygen to behave in a different manner depending upon the stoichiometry of the material. Theoretical studies that calculate the formation energy for various defects in $\mathrm{GaN}$ often provide them as a function of the Fermi level of GaN. Quite large changes in formation energy are reported for some defects as the Fermi level scans the band gap (see for example Ref. 15). Compensation and localized Coulombic effects may complicate such dependencies so that gallium-rich $\mathrm{GaN}$ must be treated uniquely. The role of oxygen in gallium-rich material appears to have a strong relationship to the defect structures present in the material.
Theoretical studies of the structure of threading dislocations have been made for gallium-rich GaN. ${ }^{16-21}$ Elsner et al. ${ }^{18}$ carried out self-consistent-charge density-functional tight-binding (SCC-DFTB) calculations looking at the role of oxygen in relation to these defects. The findings of Elsner et al. ${ }^{18}$ may be particularly relevant to understanding the results of this study. These authors assert, in a number of papers, ${ }^{18-21}$ that for Ga-rich GaN growth conditions, with sufficient available oxygen, it is energetically favorable for a gallium vacancy defect, complexed with three oxygen atoms on nitrogen sites, i.e., $V_{\mathrm{Ga}^{-}}\left(\mathrm{O}_{\mathrm{N}}\right)_{3}$, to form at threading dislocations. This defect complex is apparently very stable once formed and is electrically inactive. The associated $\left[\mathrm{V}_{\mathrm{Ga}^{-}}\right.$ $\left.\left(\mathrm{O}_{\mathrm{N}}\right)\right]^{2-}$ and $\left[\mathrm{V}_{\mathrm{Ga}^{-}}\left(\mathrm{O}_{\mathrm{N}}\right)_{2}\right]^{1-}$ complexes were also thought to form though calculations show that these are less stable and should respectively be deep double-acceptor and deep singleacceptor defects. ${ }^{18}$ Segregation of these defect complexes at dislocation sites was believed to occur because the formation energies were more favorable within the stress field of the dislocation sites than in the bulk material. ${ }^{18}$ It was further suggested that the presence of the electrically inactive $\mathrm{V}_{\mathrm{Ga}^{-}}$ $\left(\mathrm{O}_{\mathrm{N}}\right)_{3}$ complex inhibits overgrowth by $\mathrm{GaN}^{20}$ so that the defects grow parallel to the $c$ axis.

There is some experimental verification of the models proposed by Elsner et al.: Oila et $_{\text {al. }}{ }^{22}$ used low-energy positron beam analysis and secondary ion mass spectroscopy (SIMS) to study vacancy formation in $\mathrm{GaN}$ and found that the Ga vacancy concentration increased in the presence of oxygen for $n$-type GaN. This suggests that stable $\mathrm{Ga}$ vacancy-oxygen complexes may have formed. LilientalWeber et al. ${ }^{23}$ also noted an increase in the density of GaN nanotube defects with the addition of oxygen, possibly providing evidence that these complexes tend to segregate and form extended defect structures.

From the aforementioned discussions, two points of importance can be ascertained: in gallium-rich $\mathrm{GaN}$, oxygen defect complex formation with Ga vacancies may be prevalent, and that there may be a segregation of these complexes at dislocation sites. The same two points may not hold true for nitrogen-rich or extremely stoichiometric GaN. With this in mind, the experiments described next were carried out to observe any morphological changes resulting from the introduction of oxygen into gallium-rich GaN. Large amounts of oxygen have been used to exaggerate effects that would be expected when lesser amounts of oxygen are present.

\section{EXPERIMENTS AND RESULTS}

The films examined here were grown at $540{ }^{\circ} \mathrm{C}$ using the remote plasma enhanced laser induced chemical vapor deposition (RPE-LICVD) technique developed at Macquarie University and described elsewhere. ${ }^{24-26}$ Material quality from this technique is comparable to MBE grown material with unintentionally doped $n$-type material being produced with room-temperature mobilities of $100-200 \mathrm{~cm}^{2} / \mathrm{V} \mathrm{s}^{26} \mathrm{~A}$ high vacuum system was used for the film growth, and the oxygen content of the films was moderated by controlling the background pressure prior to growth. The GaN films were grown on (100) silicon substrates that had previously been de- 
TABLE I. Atomic film content determined by ERD analysis of the three $\mathrm{GaN}$ films, the background pressure of the growth rig is also indicated for each of the sample films.

\begin{tabular}{lccr}
\hline \hline & Sample A & Sample B & Sample C \\
\hline Ga at. \% & $41.3 \pm 0.4$ & $40.6 \pm 2.0$ & $47.5 \pm 2.0$ \\
$\mathrm{~N}$ at. \% & $25.8 \pm 0.3$ & $42.1 \pm 2.0$ & $43.0 \pm 2.0$ \\
$\mathrm{O}$ at. \% & $22.0 \pm 0.4$ & $9.5 \pm 1.0$ & $2.4 \pm 0.5$ \\
$\mathrm{C}$ at. \% & $2.6 \pm 0.2$ & $4.8 \pm 1.0$ & $3.9 \pm 0.5$ \\
$\mathrm{H}$ at. \% & $8.3 \pm 0.1$ & $3.0 \pm 0.5$ & $3.2 \pm 0.5$ \\
$\begin{array}{l}\text { Background } \\
\text { chamber pressure }\end{array}$ & $1 \times 10^{-4}$ Torr & $5 \times 10^{-6}$ Torr & $2 \times 10^{-6}$ Torr \\
prior to growth & & & \\
\hline \hline
\end{tabular}

greased and dip etched in $\mathrm{HF}: \mathrm{H}_{2} \mathrm{O}$ 1:10 solution, rinsed in 18 $\mathrm{M} \Omega \mathrm{cm} \mathrm{H}_{2} \mathrm{O}$ and then dried under nitrogen gas flow.

The samples were all grown under similar conditionsapart from the variation in the background vacuum level (see Table I). The set conditions produce Ga-rich material under low oxygen conditions, and a slight yellow coloration is noted for films grown under the same conditions on sapphire substrates. This yellow coloration is similar to that seen for Ga-rich bulk grown GaN. ${ }^{27} 15$ films were grown, three of which underwent ERD analysis, as outlined next. These three samples are labeled here as $\mathrm{A}, \mathrm{B}$, and $\mathrm{C}$ reflecting the decrease in oxygen content from 22 at. $\%$ to 9.5 at. $\%$ and then to 2.4 at. \%, respectively. The three samples are representative of the larger group. The morphology results for the other samples were consistent with these three and the same oxygen correlation described in this article was observed for the larger group, though the oxygen content was confirmed by less accurate energy dispersive $\mathrm{x}$-ray spectroscopy (EDS) measurements.

XRD was carried out using a Siemens D5000 X-ray Diffractometer, while the AFM results were measured using a Park Scientific Instruments AutoProbe in the contact mode. The EDS spectra (to be published elsewhere) were collected with a Philips XL30ESEM environmental scanning electron microscope (SEM).

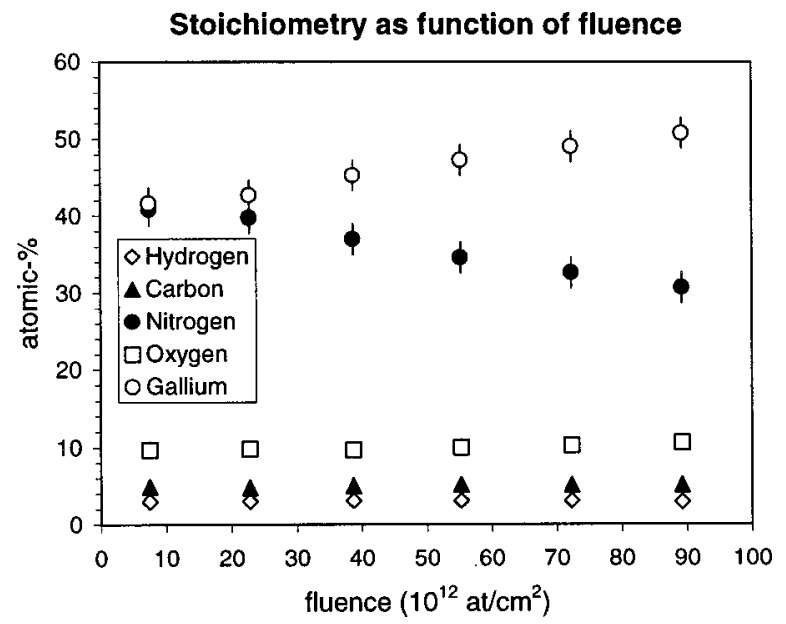

FIG. 1. Elemental content (in at. \%) as a function of beam fluence for $\mathrm{GaN}$ sample B grown on (100) Si. The sample has 9.5 at. \% oxygen.
After growth, the three sample films, A, B, and C, were submitted to the ANU where a unique system for ERD analysis using heavy ion beams is available. This system is particularly suited to the detection and quantitative analysis of light elements such as nitrogen, oxygen, and hydrogen. We were interested in the use of this system because of its high detection efficiency and the promise of lower ion damage during beam exposure. Previous experience with a different type of heavy ion ERD setup, with lower detection efficiency, had resulted in severe nitrogen loss under heavy ion beam bombardment so that an accurate compositional analysis could not be carried out.

For the ERD measurements, a beam of $200 \mathrm{MeV}{ }^{197} \mathrm{Au}$ ions from the ANU 14UD Pelletron accelerator was used. Recoil ions, including hydrogen, were detected at $45^{\circ}$ relative to the beam direction using a large detection solid angle $(3.5 \mathrm{~ms})$ gas ionization detector. The angle between the sample normal and beam direction was $112.5^{\circ}$. Kinematic energy broadening over the acceptance angle was corrected using the position information provided by the detector. ${ }^{7}$ For sample A, the film/substrate interface was well resolved so that the film composition could be determined directly with excellent accuracy by normalizing the detected recoil yields with the Rutherford scattering cross section. The film/ substrate interface was not resolved for samples B and C, since the films were thicker than the probing depth of the technique, which is $\sim 10^{19}$ atoms $/ \mathrm{cm}^{2}$ for this material. A direct comparison of the yields was thus not possible. However, film compositions have been determined by adapting simulations to the measured energy spectra. The hydrogen spectra for the films were analyzed by comparing them with that obtained for a Kapton film with constant $\mathrm{H}$ content. ${ }^{28}$

An example of the ERD results is shown in Fig. 1 for sample B, a breakdown of the sample compositions is also shown in Table I. For all three films, the stoichiometry changes linearly with the fluence of incident ${ }^{197} \mathrm{Au}$ ions, which can be attributed to the beam induced desorption of the volatile elements nitrogen and hydrogen. The initial composition of the films was therefore determined by linear extrapolation to zero-ion fluence.

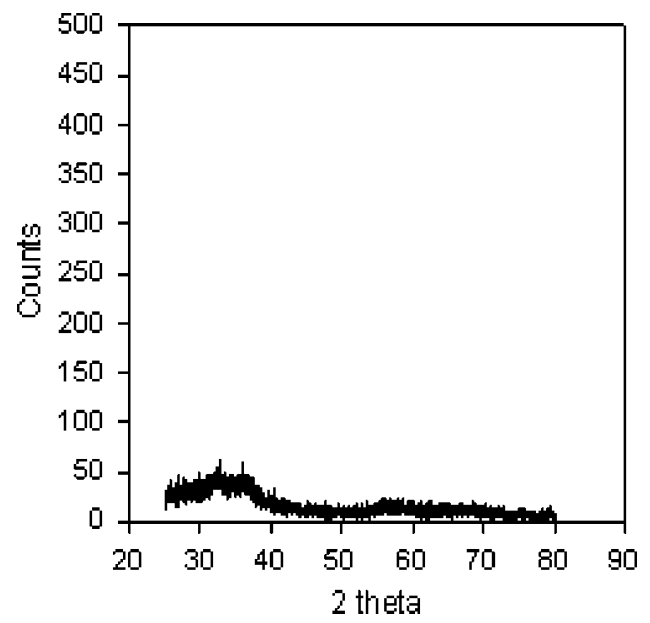

FIG. 2. XRD spectrum for GaN sample A. 


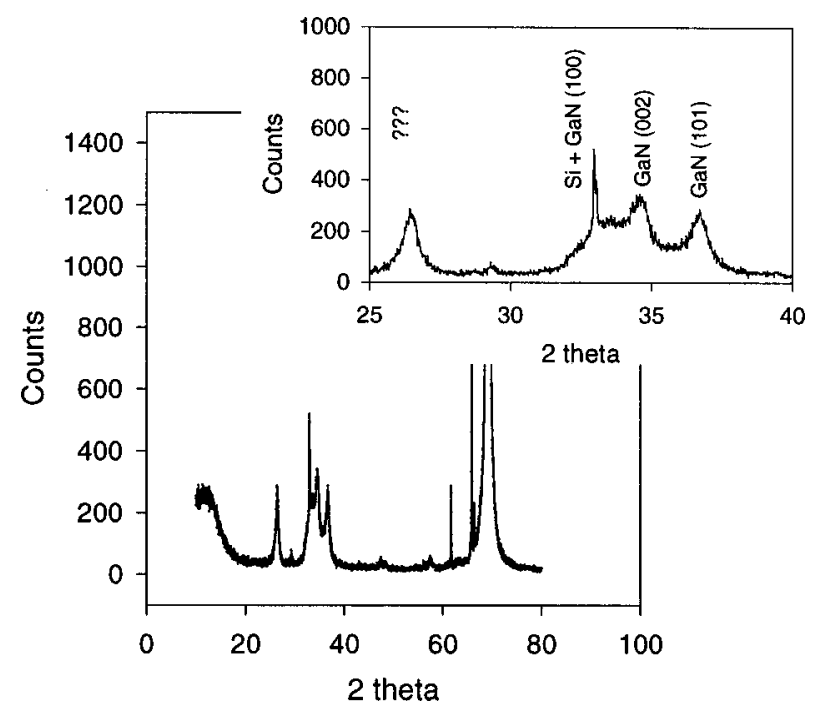

FIG. 3. XRD spectrum for GaN sample B. The inset shows a smaller $2 \theta$ region where the $\mathrm{GaN}$ peaks dominate.

The XRD and AFM results, for the samples are shown in Figs. 2-4 and Figs. 5-7, respectively. The two small peaks in Fig. 2, at 32.2 and $36.12 \theta$ degrees are, respectively attributed to the $(10 \overline{1} 0)$ and $(10 \overline{1} 1)$ reflections for GaN. Their small size (a $3 \mathrm{~h}$ data collection period was used for all the XRD spectra) and broadness indicates that the film is composed of small crystallites.

The XRD spectrum of sample B is shown in Fig. 3. A narrower spectral range is shown in the inset of Fig. 3 for this sample, since some interference from Si peaks is evident at the higher peak angles and the major peaks of interest are all in one area. In fact, a small $\mathrm{Si}$ substrate peak is still present on top of a $\mathrm{GaN}$ peak at $32.962 \theta$ degrees, recognizable by its narrowness compared to the $\mathrm{GaN}$ peaks. This sample shows several prominent gallium nitride related peaks at 33.0, 34.6, and $36.72 \theta$ degrees. The presence of these multiple $\mathrm{GaN}$ reflections indicates polycrystalline material with multiple-crystal orientation. Another unknown

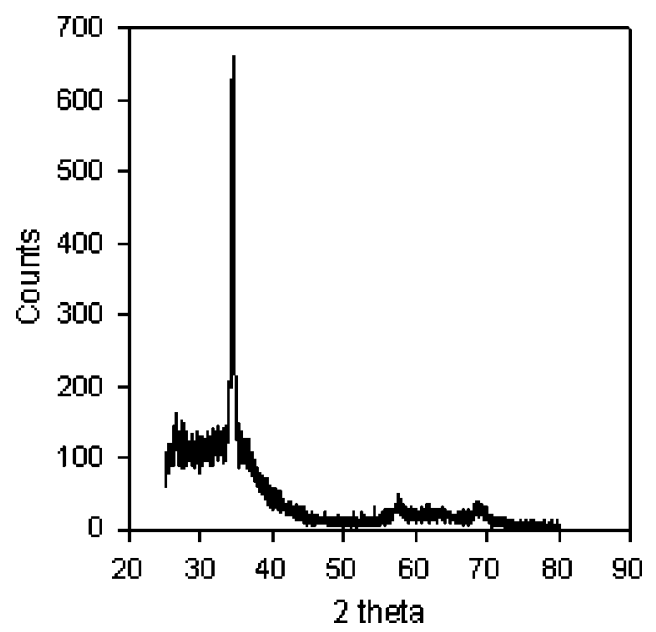

FIG. 4. XRD spectrum for GaN sample C. The spectrum shows a predominant (0002) peak indicating that the $c$ axis is oriented perpendicular to the surface of the substrate.

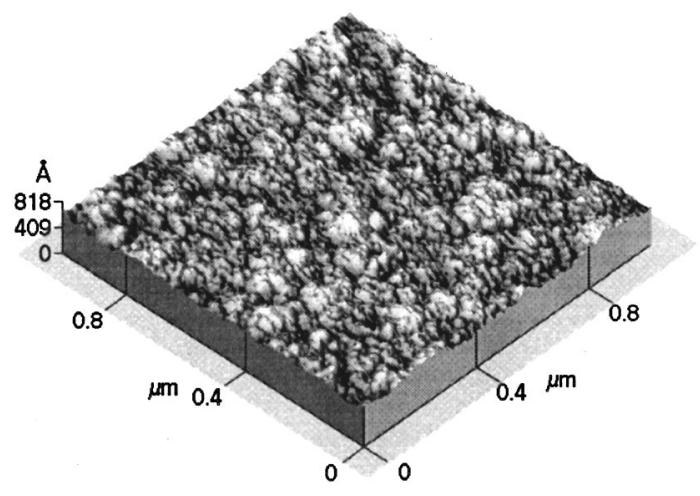

FIG. 5. AFM surface map of GaN sample A.

peak is present at a lower angle (26.45 $2 \theta$ degrees) and may be due to the incorporated oxygen. A cross-sectional SEM image taken with a 6300 JOEL field-emission gun (FEG) SEM confirms the polycrystallinity of the sample (Fig. 8).

For sample C, the XRD spectrum of Fig. 4 shows that the multiorientation observed for sample B has largely disappeared. A single strong peak is evident at $34.42 \theta$ degrees, which is the (0002) reflection of GaN. The presence of this reflection indicates predominantly (0001) oriented material with the hexagonal structure $c$ axis aligned normal to the (100) cubic silicon surface. A FEG-SEM cross section of a film with a slightly higher oxygen content, shown in Fig. 9, confirms this. Some small GaN peaks at 57.7 and $68.62 \theta$ degrees indicate the presence of a small amount of crystal multiorientation, although the (0001) orientation clearly dominates. The SEM micrograph shows that this multiorientation originates from an incomplete columinar structure. The unknown peak at $26.452 \theta$ degrees, most probably a gallium-nitrogen-oxygen compound is also still present in this spectrum, though again at a much lower intensity than the (0002) reflection.

The AFM measurements show the morphology of the sample surface, for the same samples examined with XRD. Figure 5 shows the AFM three-dimensional topology of sample A. The sample appears to show many small crystallites of approximately $25 \mathrm{~nm}$ average diameter. In Fig. 6, the topology of sample B, with lower oxygen is shown. For this sample, there is an appreciable increase in the crystal size. The multiple-crystal orientation evident in the XRD spec-

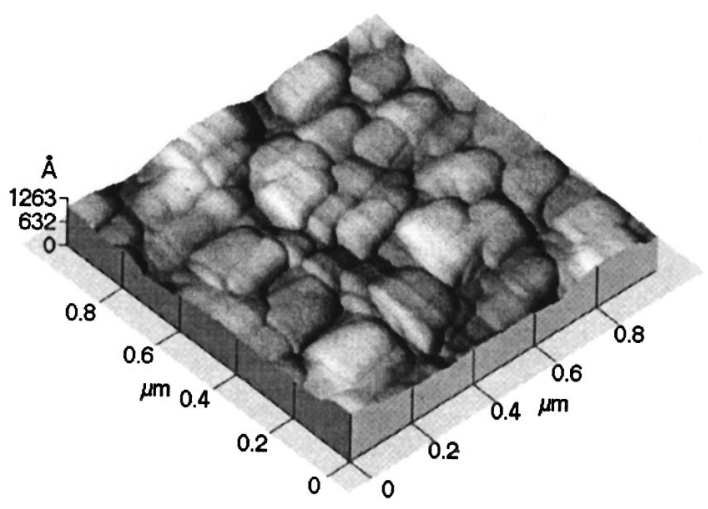

FIG. 6. AFM surface map of GaN sample B. 


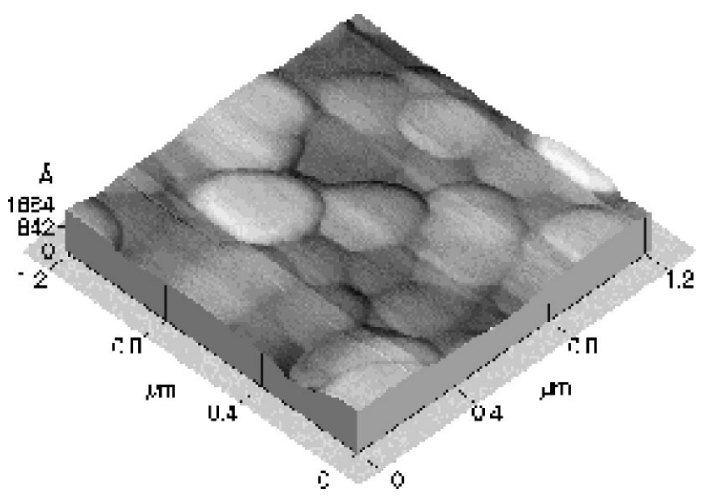

FIG. 7. AFM surface map of GaN sample C.

trum for sample B is also evident in the AFM topology with some crystallites having their $c$ axis parallel to the crystal surface.

Figure 6 shows a three-dimensional topological image of sample C. Again with the reduction in oxygen content, down to 2.4 at. \% for this sample, there has been an increase in crystal size to an average of approximately $246 \mathrm{~nm}$. The multiorientations present for sample B with higher oxygen content have disappeared and the surface appears to have fairly uniform crystallinity. This is in accord with the XRD results, which show a single strong XRD peak indicative of a single-dominant crystal orientation.

\section{DISCUSSION}

For the GaN films examined here, the aforementioned data show an increase in crystal size with lower oxygen content. We suggest that this might be due to the segregation of

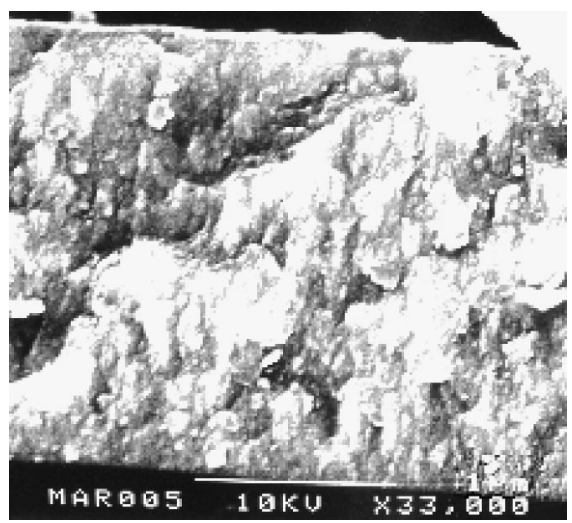

FIG. 8. Film cross section for sample B taken using a FEG-SEM. Small crystallites are clearly visible.

oxygen at the crystal boundaries. A similar model of oxygen segregation at the grain boundaries has been proposed for polycrystalline $\mathrm{AlN},{ }^{29}$ which is chemically similar to GaN but, in that case, it was suggested that the oxygen was incorporated as a result of postgrowth ex situ exposure to air. In our case, the oxygen incorporation was predominantly during growth, as controlled by the background ambient pressure of the chamber (see Table I). Our suggestion of oxygen segregation during growth is supported by the work of Elsner et al. ${ }^{18-21}$ discussed in Sec. II, who were able to show that the segregation of oxygen by the formation of $V_{\mathrm{Ga}^{-}}\left(\mathrm{O}_{\mathrm{N}}\right)_{n}$ complexes is energetically favorable. They further suggested that the formation of these complexes inhibited any overgrowth by $\mathrm{GaN}$ so that segregated collections of the complexes extended to the film surface as dislocations running parallel to the $c$ axis of the film. Our results may be seen as

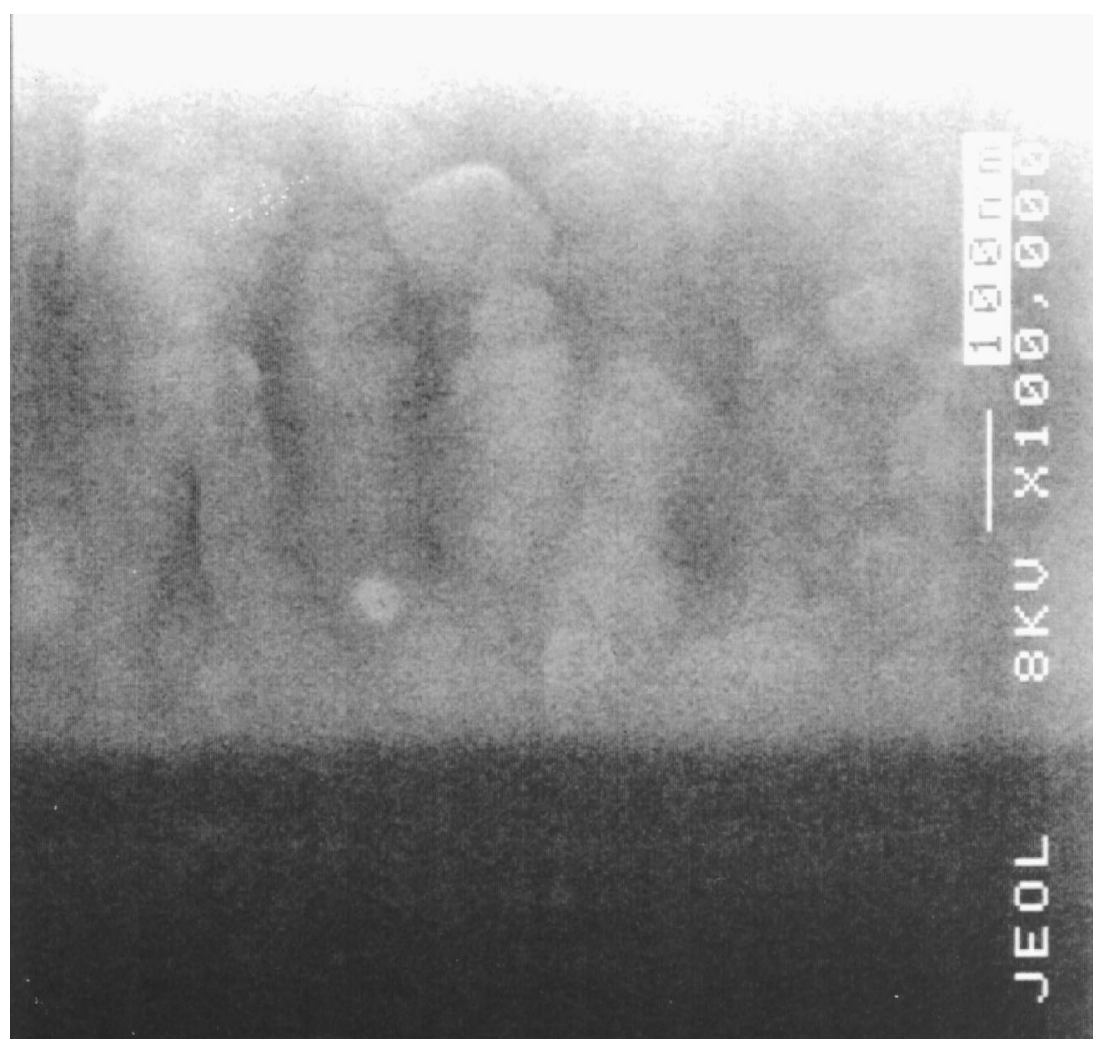

FIG. 9. Film cross section of a sample with oxygen content intermediary between samples B and C. $c$-axis oriented crystallites are visible, though the columinar structure is incompletely developed for this example. 
partial confirmation of the theoretical work of Elsner et al., except that here quite large amounts of oxygen have resulted in segregation at polycrystalline boundaries and not just at threading dislocations. The greater amount of oxygen present has allowed the coalescence of dislocations. With less oxygen, present fewer grain boundaries result and larger crystallites are evident. Presumably, the presence of even smaller amounts of oxygen than that studied here would see a return to dislocation defects in place of grain boundaries (in the absence of film strain related to the substrate-film lattice mismatch). Sufficient adatom mobility must of course be present at the growth temperature for oxygen to migrate to a suitable lattice site.

Direct evidence of this model, by elemental mapping, would be difficult to obtain considering the dimensions of the crystals and the spatial resolution required to distinguish oxygen at the grain boundary. However, if the model holds true, there should be some relationship between the surface areas of the crystals and the total oxygen content of the film. Therefore, in order to confirm the model, we have carried out some simplified calculations of the expected oxygen content of the films based on the available intergranular surface area, and then checked this against the known oxygen content found by the ERD analysis.

An approximate calculation can be carried out in the following manner. For sample $\mathrm{C}$, assuming $c$-axis oriented crystals that are continuous through the thickness of the film, but have an " $a$ "-axis diameter $(D)$ of $246 \mathrm{~nm}$, then the volume and available surface area of the crystals (excluding the top and bottom surfaces of the crystals, since only the bulk is of interest) can be found and used for the calculation. The crystal volume can be divided by the number of unit cells for the hexagonal GaN structure using the lattice constants ${ }^{30} a$ $=3.186 \AA$ and $c=5.178 \AA$. The volume $(V)$ of the unit cell is given by

$$
V=\frac{a^{2} c \sqrt{3}}{2},
$$

where there are two GaN molecules per unit cell volume. The number of unit cells $(N)$ that can be accommodated in our crystal volume is, therefore, given by

$$
N=L \cdot \pi \cdot(D / 2)^{2} / V,=5.41 \times 10^{5},
$$

where $L$ is the height of the crystals, in this case, for simplicity, taken to be the unit crystal height equal to " $c$ ". This yields a total of $1.08 \times 10^{6}$ gallium atoms, since there are two gallium atoms per unit cell. There is also the same number of nitrogen atoms in our crystal volume. The minimum number of unit cells $\left(N_{S}\right)$ at the crystal surface (excluding the top and bottom surfaces of the crystal which are at the film surface and not representative of the bulk oxygen) is given by

$$
N_{S}=D \cdot \pi \cdot / a, \quad=2505 .
$$

Assuming the crystal surfaces are terminated by the oxygen related $V_{\mathrm{Ga}^{-}}\left(\mathrm{O}_{\mathrm{N}}\right)_{3}$ complex mentioned previously, then for each of the two gallium atoms missing as vacancies from a unit cell, we should expect six related oxygen atoms. This is the case regardless of whether or not all the oxygen atoms are contained in the unit cell. Three separate oxygen atoms are required for each vacancy in order to maintain charge neutrality. This gives us a total of 15030 oxygen atoms at the surface of our crystal, therefore, as an atomic percentage of composition, the total oxygen content on the outside of the crystal (in the bulk) can be found by the following calculation

$$
\text { at. } \begin{aligned}
\% \text { oxygen } & =100\left(6 N_{S} /\left(4 N-2 N_{S}\right)\right), \\
& =100\left(15030 /\left(4 \times 5.41 \times 10^{5}-2 \times 2505\right)\right) \\
& =0.70 \text { at. } \%,
\end{aligned}
$$

where the second term in the denominator of Eq. (4) takes into account the gallium vacancies present at the crystal surface. Doubling this figure to take into account the oxygen termination on adjoining crystals gives a total oxygen content of 1.4 at. \% for the film. This value agrees reasonably well with the oxygen content of sample $\mathrm{C}$ given by the ERD results (see Table I), and for which the AFM results indicate an average crystal diameter of approximately $246 \mathrm{~nm}$.

A similar calculation can be performed for sample B, however for sample B, the crystals do not extend the length of the film. The oxygen content must, therefore, be calculated for the total surface area of a typical crystal. Taking an idealized typical crystal of cylindrical volume, with $50 \mathrm{~nm}$ diameter $(D)$ and $150 \mathrm{~nm}$ length $(L)$ - as estimated from the AFM results - then a total oxygen content of 8.8 at. \% can be calculated for the film. Given the inaccuracies involved in this approximate calculation, and the error present for the ERD analysis, the agreement between this calculated value and the ERD results (see Table I) is excellent.

A similar calculation was made for sample A, and assuming $25 \mu \mathrm{m}$ diameter $25 \mu \mathrm{m}$ length cylindrical crystals (again estimated from the AFM results) yielded a film with a 26.8 at. \% oxygen content. This is again in reasonable agreement with the ERD analysis data of Table I.

From the approximate calculations made herein, we can see that the available surface area at the polycrystalline boundaries can indeed accommodate a significant portion of the oxygen in these gallium-rich GaN films. Formation of the $V_{\mathrm{Ga}^{-}}\left(\mathrm{O}_{\mathrm{N}}\right)_{3}$ defect complex, which previous calculations by Elsner et al. ${ }^{18-21}$ have shown to be energetically stable, to be electrically neutral, and to segregate at defect sites, is indicated by the present calculations since this defect complex provides adequate oxygen sites at the crystal surface to account for the measured values of oxygen content of the film. The incorporation of oxygen during growth, therefore, appears to have a strong influence on the size of crystals for $\mathrm{GaN}$. Given that the oxygen segregates at grain boundaries, and if substrates with low lattice mismatch or appropriate buffer layers are available, then lowering the oxygen content of the films should result in larger crystals or even singlecrystal material at low growth temperatures. For the material grown here on silicon, however, it is expected that only a moderate gain in crystal size can be obtained by the further lowering of the oxygen content of the film, as the lattice mismatch between pure $\mathrm{GaN}$ and $\mathrm{Si}$ is large at $20 \% .^{31}$

Though the ERD results show nonnegligible amounts, the role of hydrogen and carbon has been ignored in the previous discussion. It is probable that a considerable portion 
of the carbon and hydrogen is adventitious with these smaller atoms being absorbed from the atmosphere through voids present at the grain boundaries. Certainly, hydrogen is known to be present at percentage levels in many forms of $\mathrm{GaN}$ and is easily absorbed under many conditions. ${ }^{9}$

The 2.4 at. \% oxygen level of sample $\mathrm{C}$ represents the lowest oxygen incorporation level for the samples grown by RPE-LICVD for this study. Despite this level of oxygen incorporation, good quality polycrystalline material has been realized, as discussed briefly in Sec. III. The formation of an electrically neutral $V_{\mathrm{Ga}}-\left(\mathrm{O}_{\mathrm{N}}\right)_{3}$ defect complex would explain the reasonable mobilities obtained for this material, at moderate carrier concentration, in the presence of such high oxygen levels.

\section{CONCLUSIONS}

ERD measurements of film oxygen composition have been shown to be correlated with crystal size for gallium-rich $\mathrm{GaN}$. Larger crystal sizes are achieved with reduced oxygen, as controlled in this case, by the background vacuum level. In agreement with the work of Elsner et al. ${ }^{18-21}$ the oxygen is believed to segregate at defect sites, predominantly as $V_{\mathrm{Ga}}-\left(\mathrm{O}_{\mathrm{N}}\right)_{3}$ defect complexes. In this case, however, the defect sites are crystalline boundaries. A comparison of measured oxygen content with the calculated available surface area of crystals in the polycrystalline GaN films has shown that a significant proportion of the available oxygen can be accommodated at the grain boundaries. The $\mathrm{GaN}$ was grown in a temperature range $\left(\sim 540^{\circ} \mathrm{C}\right)$ where it is expected to be gallium rich. This is believed to be an important aspect of this type of oxygen incorporation. There is, at present, no evidence to suggest that similar segregation should occur for stoichiometric or nitrogen-rich GaN.

XRD and AFM results were used to provide the morphological data required for this study while a low damage ERD system was used to determine the atomic composition of the GaN with its oxygen, hydrogen, and carbon impurities.

\section{ACKNOWLEDGMENTS}

The authors would like to acknowledge the support of a U. S. NICOP Contract, No. N00014-99-1-GO17 sponsored through the U. S. Office of Naval Research. One of the authors (K.S.A.B.) would like to further acknowledge the support of a Macquarie University Research Fellowship.

${ }^{1}$ J. W. Orton and C. T. Foxon, Rep. Prog. Phys. 61, 1 (1998).

${ }^{2}$ D. P. Bour, N. M. Nickel, C. G. Van de Walle, M. S. Kneissl, B. S. Krusor, Ping Mei and N. M. Johnson, Appl. Phys. Lett. 76, 2182 (2000).
${ }^{3}$ S. Guha, N. A. Bojarczuk, and D. W. Kisker, Appl. Phys. Lett. 69, 2879 (1996).

${ }^{4}$ K. R. Evans, T. Lei, and C. R. Jones, Solid-State Electron. 41, 339 (1997).

${ }^{5}$ A. V. Blant, S. V. Novikov, T. S. Cheng, L. B. Flannery, I. Harrison, R. P. Campion, D. Korakakis, E. C. Larkins, Y. Kribes, and C. T. Foxon, J. Cryst. Growth 203, 349 (1999).

${ }^{6}$ H. Timmers, R. G. Elliman, G. R. Palmer, T. R. Ophel, and D. J. O'Connor, Nucl. Instrum. Methods Phys. Res. B 136, 611 (1998).

${ }^{7}$ H. Timmers, T. R. Ophel, and R. G. Elliman, Nucl. Instrum. Methods Phys. Res. B 161, 19 (2000).

${ }^{8}$ H. Timmers, R. G. Elliman, and T. R. Ophel, Nucl. Instrum. Methods Phys. Res. A 447, 536 (2000).

${ }^{9}$ S. J. Pearton, J. C. Zolper, R. J. Shul, and F. Re, J. Appl. Phys. 86, 1 (1999).

${ }^{10}$ O. Ambacher, J. Phys. D 31, 2653 (1998).

${ }^{11}$ H. Ch. Alt, Mater. Sci. Forum 83, 369 (1992).

${ }^{12}$ K. S. A. Butcher, L. Mo, D. Alexiev, and T. L. Tansley, J. Cryst. Growth 156, 361 (1995)

${ }^{13}$ K. S. A. Butcher, D. Alexiev, and T. L. Tansley, Aust. J. Phys. 46, 317 (1993).

${ }^{14}$ Y. Kim, S. G. Subramanya, H. Siegle, J. Kruger, P. Perlin, E. R. Weber, S. Ruvimov, and Z. Liliental-Weber, J. Appl. Phys. 88, 6032 (2000).

${ }^{15}$ C. G. Van de Walle, J. Neugebauer, and C. Stampfl, in Gallium Nitride and Related Semiconductors, edited by J. H. Edgar, S. Strite, I. Akasaki, H. Amano, and C. Wetzel (Inspec, London, 1999), p. 281.

${ }^{16}$ K. Leung, A. F. Wright, and E. B. Stechel, Appl. Phys. Lett. 74, 2495 (1999).

${ }^{17}$ S. M. Lee, M. A. Belkhir, X. Y. Zhu, Y. H. Lee, Y. G. Hwang, and T. Frauenheim, Phys. Rev. B 61, 16033 (2000).

${ }^{18}$ J. Elsner, R. Jones, M. I. Heggie, P. K. Sitch, M. Haugk, T. Frauenheim, S. Oberg, and P. R. Briddon, Phys. Rev. B 58, 12571 (1998).

${ }^{19}$ J. Elsner, R. Jones, M. Haugk, R. Gutierrez, T. Frauenheim, M. I. Heggie, S. Oberg, and P. R. Briddon, Appl. Phys. Lett. 73, 3530 (1998).

${ }^{20}$ R. Gutierrez, M. Haugk, T. Frauenheim, J. Elsner, R. Jones, M. I. Heggie, S. Oberg, and P. R. Briddon, Philos. Mag. Lett. 79, 147 (1999).

${ }^{21}$ A. T. Blumenau, J. Elsner, R. Jones, M. I. Heggie, S. Oberg, T. Frauenheim, and P. R. Briddon, J. Phys.: Condens. Matter 12, 10223 (2000).

${ }^{22}$ J. Oila, V. Ranki, J. Kovioja, K. Saarinen, P. Hautojarvi, J. Likonen, J. N. Baranowski, K. Pakula, T. Suski, M. Leszczynski, and I. Grzegory, Phys. Rev. B 63, 045205 (2001).

${ }^{23}$ Z. Lilental-Weber, Y. Chen, S. Ruvimov, and J. Washburn, Phys. Rev. Lett. 79, 2835 (1997)

${ }^{24}$ B. Zhou, X. Li, T. L. Tansley, K. S. A. Butcher, and M. R. Phillips, J. Cryst. Growth 151, 249 (1995)

${ }^{25}$ B. Zhou, X. Li, T. L. Tansley, and K. S. A. Butcher, J. Cryst. Growth 160, 201 (1996).

${ }^{26}$ B. Zhou, X. Li, T. L. Tansley, and K. S. A. Butcher, Appl. Surf. Sci. 100, 643 (1996).

${ }^{27} \mathrm{Z}$. Liliental-Weber et al., Proceedings of the Topical Workshop on III-V Nitrides (TWN95) edited by I. Akasaki and K. Onabe (Elsevier Science, New York, 1997), p. 167.

${ }^{28}$ R. G. Elliman, H. Timmers, T. R. Ophel, T. D. M. Weijers, L. S. Wielunski, and G. L. Harding, Nucl. Instrum. Methods Phys. Res. B 161, 231 (2000).

${ }^{29}$ M. Kishi, M. Suzuki, and K. Ogawa, Jpn. J. Appl. Phys., Part 1 31, 1153 (1992).

${ }^{30}$ Taken from the JCPDS-ICDD PDF-2 database.

${ }^{31}$ H. Okumura, in Gallium Nitride and Related Semiconductors, edited by J. H. Edgar, S. Strite, I. Akasaki, H. Amano, and C. Wetzel (Inspe, London, 1999), p. 402. 